\title{
Salicylaldoxime derivatives as new leads for the development of carbonic anhydrase inhibitors
}

\author{
Tiziano Tuccinardi ${ }^{\mathrm{a}, *}$, Simone Bertini ${ }^{\mathrm{a}}$, Carlotta Granchi ${ }^{\mathrm{a}}$, Gabriella Ortore ${ }^{\mathrm{a}}$, Marco Macchia ${ }^{\mathrm{a}}$, \\ Filippo Minutolo ${ }^{\mathrm{a}}$, Adriano Martinelli ${ }^{\mathrm{a}}$, Claudiu T. Supuran ${ }^{\mathrm{b}}$ \\ a Dipartimento di Scienze Farmaceutiche, Università degli Studi di Pisa, Via Bonanno 6, 56126 Pisa, Italy \\ ${ }^{\mathrm{b}}$ Polo Scientifico, Laboratorio di Chimica Bioinorganica, Room 188, Università degli Studi di Firenze, Via della Lastruccia 3 , 50019 Sesto Fiorentino, Florence, Italy
}

\section{A R T I C L E I N F O}

\section{Article history:}

Available online 7 September 2012

\section{Keywords:}

Carbonic anhydrase

Salicylaldoxime

Docking

QM/MM calculation

Zinc binding group

\begin{abstract}
A B S T R A C T
New compounds containing a novel zinc binding group (salicylaldoxime system) were identified as effective inhibitors of carbonic anhydrases (CAs). This structural motif seems to bind the catalytic zinc ion of CAs, revealing itself as a new valid alternative to the sulfonamide group. Computational procedures were used to investigate the binding mode of this class of compounds, within the active site of CAII. This study suggests that the salicylaldoxime moiety binds the zinc ion through the oxime oxygen atom that also forms an H-bond with T199. The results herein obtained will allow the development of new CA-inhibitors bearing the salicylaldoxime moiety.
\end{abstract}

(c) 2012 Elsevier Ltd. All rights reserved.

\section{Introduction}

Carbonic anhydrases (CAs) are widespread, distributed zinc metalloenzymes. They belong to a superfamily of ubiquitous metalloproteins and catalyze the reversible hydration of $\mathrm{CO}_{2}$ to form $\mathrm{HCO}_{3}{ }^{-}$, which is involved in many biosynthetic reactions, among which are gluconeogenesis, lipogenesis, the synthesis of some amino acids, and pyrimidine nucleotide biosynthesis. Moreover, these enzymes are involved in $\mathrm{pH}$ homeostasis, bone resorption, ion transport, electrolyte secretion in a variety of tissues, calcification, and tumorigenesis. ${ }^{1}$ The metal ion is critical for catalysis, as the apoenzyme is devoid of any catalytic activity. ${ }^{2}$

In mammals, 16 CA isozymes or CA-related proteins have been identified and mainly differ in their subcellular localization, catalytic activity and tissue distribution. Five CAs are cytosolic (CAI-III, VII, and XIII), two are mitochondrial (CAVA and VB), one is secreted (CAVI), and the others are membrane-bound (CAIV, IX, XII, XIV and XV). ${ }^{3}$ Many CA subtypes constitute interesting targets for the design of pharmacological agents useful for the treatment of various pathologies such as glaucoma, neurological disorders, obesity, cancer, epilepsy and osteoporosis. ${ }^{4}$

Classical CA inhibitors are constituted by primary sulfonamides, which coordinate the zinc ion with their terminal deprotonated nitrogen atom and form hydrogen bonds with Thr199. The side effects often associated to the use of sulfonamide derivatives have led to the development of new zinc binding groups (ZBGs). ${ }^{5}$ To

\footnotetext{
* Corresponding author. Tel.: +39 050 2219595; fax: +39 0502219595 .

E-mail address: tuccinardi@farm.unipi.it (T. Tuccinardi).
}

date, sulfamates, sulfamides, phenols ${ }^{6}$ and polyamines ${ }^{7}$ have been reported to be efficient ZBGs; furthermore, very recent studies on matrix metalloproteinase inhibitors by Klebe and co-workers found four new CA ZBGs with micromolar activities and different zinc coordination geometries. ${ }^{5}$ Here we report another CA ZBG, discovered starting from known estrogen receptor modulators, constituted by the salicylaldoxime moiety. ${ }^{8}$ The experimental studies were developed together with in silico techniques, aimed to propose a reliable binding disposition for this new class of CA inhibitors.

\section{Results and discussion}

\subsection{CA inhibition studies}

Starting from our library of estrogen receptor ligands, ${ }^{9-12}$ we selected, as a preliminary experimental analysis, eight candidates characterized by the presence of a salicylaldoxime system substituted with different aryl and halogen atoms (see Table 1). We selected these compounds for investigating the possible interaction of the salicylaldoxime system with the zinc ion.

The inhibition data for compounds 1-8 against four CA isoforms (hCAI, II, IX, XII) are listed in Table 1. 3,5-Difluorophenol was added as a reference compound. All the analyzed compounds showed inhibition potencies $\left(K_{\mathrm{i}}\right)$ against the tested isoforms in the range of $2.3-10.2 \mu \mathrm{M}$.

All the tested compounds showed very similar inhibition activities, thus suggesting that the analyzed substitutions did not affect the interactions of the ligand with the enzyme. The analyzed 
Table 1

Inhibition data for compounds 1-9.<smiles>COc1ccc(-c2ccc3c(c2)C=[N+](O)NO3)cc1F</smiles><smiles>CC1=C(C)N(O)COc2c1ccc(-c1ccc(O)c(F)c1)c2Cl</smiles><smiles></smiles>
7

\begin{tabular}{|c|c|c|c|c|}
\hline \multirow[t]{2}{*}{ Compound } & \multicolumn{4}{|c|}{$K_{\mathrm{i}}(\mu \mathrm{M})^{\mathrm{a}}$} \\
\hline & hCAI & hCAII & hCAIX & hCAXII \\
\hline 1 & 7.27 & 3.74 & 7.04 & 5.53 \\
\hline 2 & 3.98 & 2.30 & 6.02 & 4.31 \\
\hline 3 & 9.13 & 4.13 & 6.99 & 4.24 \\
\hline 4 & 6.93 & 3.66 & 10.19 & 5.94 \\
\hline 5 & 7.57 & 4.96 & 7.08 & 4.77 \\
\hline 6 & 6.90 & 3.32 & 10.18 & 4.53 \\
\hline 7 & 7.88 & 6.35 & 6.43 & 7.45 \\
\hline 8 & 7.65 & 5.87 & 7.72 & 3.14 \\
\hline 3,5-Difluorophenol & 38.81 & 339.79 & 0.944 & 70.22 \\
\hline
\end{tabular}

a Mean from 3 different assays. Errors were in the range of $\pm 5 \%$ of the reported values.

compounds supposedly interact with the zinc ion through either the salicylaldoxime system, or the 4-hydroxyphenyl substituent. However, the generally good inhibition potency of compound $\mathbf{4}$, that differs from the other compounds for the replacement of the $p$-hydroxy with a $p$-methoxy group in the aryl substituent, strongly support the interaction of the salicylaldoxime portion with the zinc ion.

\subsection{Docking studies}

A knowledge of the binding mode of these compounds within CAs is of recognized importance for improving the design of new CA inhibitors. To achieve this goal, the existence of an inhibitorenzyme complex model may greatly facilitate computer-aided structure optimization by using a scaffold-constrain docking procedure. ${ }^{13}$ These studies showed that compounds 1-8 efficiently bind CAs, where the salicylaldoxime system was expected to play the role of ZBG. The most accurate way to get information on the inhibitor-enzyme binding mode is undoubtedly by X-ray crystallographic studies of the corresponding complexes. However, in the

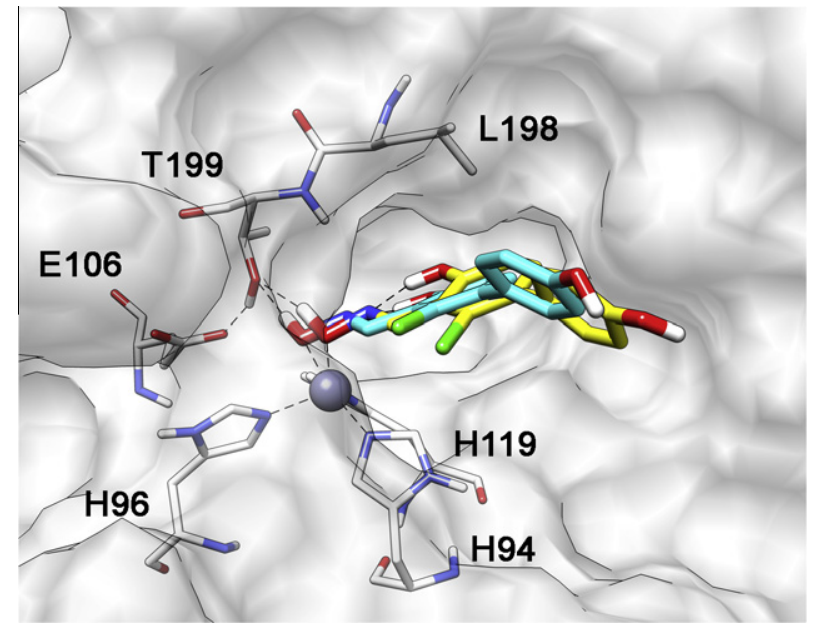

Figure 1. Superimposition of the compound $\mathbf{2}$ best docking poses into CAII, considering the catalytic zinc ion with a tetrahedral (colored yellow) or a trigonal bipyramidal (colored light blue) coordination geometry.

absence of these important experimental evidences, theoretical approaches have been widely accepted as secondary tools to produce reliable results. With this aim, we focused our attention on the binding geometry of this new ZBG (in particular, compound 2 with CAII, since this combination displayed the lowest $K_{\mathrm{i}}$ value) and carried out a series of computational approaches based on docking quantum mechanical (QM), and hybrid quantum mechanical/ molecular mechanical (QM/MM) calculations. Although docking calculations had already been successfully applied to CA inhibitors, the coordination of the reported ZBG with CAs had never been predicted before. Therefore, to support the reliability of our docking calculations and to have a deeper analysis of the ZBG interactions, both $\mathrm{QM}$ and $\mathrm{QM} / \mathrm{MM}$ approaches were applied to the docking results.

Docking of compound 2 into CAII was conducted to provide possible binding hypotheses of the ZBG. GOLD software was used for this purpose, as it proved to be a good method for docking CA inhibitors. ${ }^{14,15}$ As reported in detail in the Experimental Section, two docking runs were carried out, considering the catalytic zinc ion either with a tetrahedral, or a trigonal bipyramidal coordination geometry. The two docking runs converged in a unique ligand binding mode (see Fig. 1) with the salicylaldoxime group coordinating the zinc ion through the oxime oxygen atom that also formed an H-bond with T199. The remaining part of compound 2 did not show important additional interactions, with the exception of the aromatic ring of the salicylaldoxime system, which interacts with L198, showing a disposition similar to that of the aromatic ring of other known arylsulfonamide CA inhibitors. ${ }^{16}$

\subsection{QM studies}

To confirm the reliability of the docking results, QM optimization of the interaction between the ZBG and CAII was carried out. The catalytic zinc binding region was taken from the X-ray crystal structure (3V7X PDB code ${ }^{16}$ ) and the ligand coordinates were retrieved from the docking results. Simplifications on the binding site were made, and only the main atoms around the ZBG were included, while compound $\mathbf{2}$ was replaced by 2-chloro-salicylaldoxime as a simpler model. The complex was submitted to purely quantum mechanics optimization, using Gaussian software, with the B3LYP chemical model and the LANL2DZ and the 6$31++G(d, p)$ basis set. The QM optimization converged into solutions that are very similar to the starting docking geometry. As 

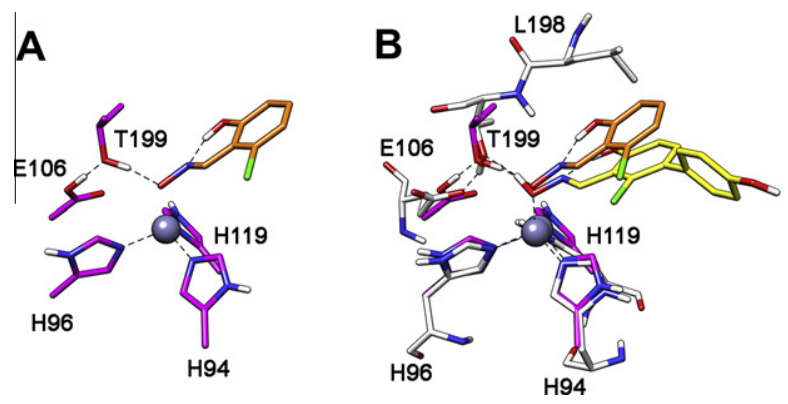

Figure 2. Final structure of the $Q M$ optimization $(6-31++G(d, p)$ basis set) and superimposition with the docking results for compound $\mathbf{2}$.

shown in Figure 2, the most important differences concerned the H-bond network: both QM optimizations determined the deprotonation of the oxime oxygen with a shift of the hydrogen from the ligand to T199, and in this conditions E106 appears to be protonated by the starting hydrogen of T199.

\subsection{QM/MM ONIOM optimization}

In order to further confirm the obtained geometries and to further analyze the ligand-protein H-bond network a QM/MM ONIOM approach ${ }^{17}$ was applied to the starting docking complex. Using this method, the molecular segment with the highest interest, in terms of binding interaction, was quantum mechanically treated, while the rest of the system was treated by means of classical mechanics, thus saving considerable computational expenses. The CAII-2 complex was divided into two layers; the first layer corresponded to the fragments previously analysed by the QM approach and the rest of the system was considered as the second layer. Two calculations were carried out, for both calculations the second layer was analysed using the MM theory and the AMBER force field whereas the first layer was analysed using the B3LYP chemical model and both the LANL2DZ and the $6-31++G(d, p)$ basis set. Both $Q M / M M$ calculations converged to a unique solution that was very similar to that obtained using the QM approach. In particular, as shown in Figure 3, the salicylaldoxamide maintained the coordination

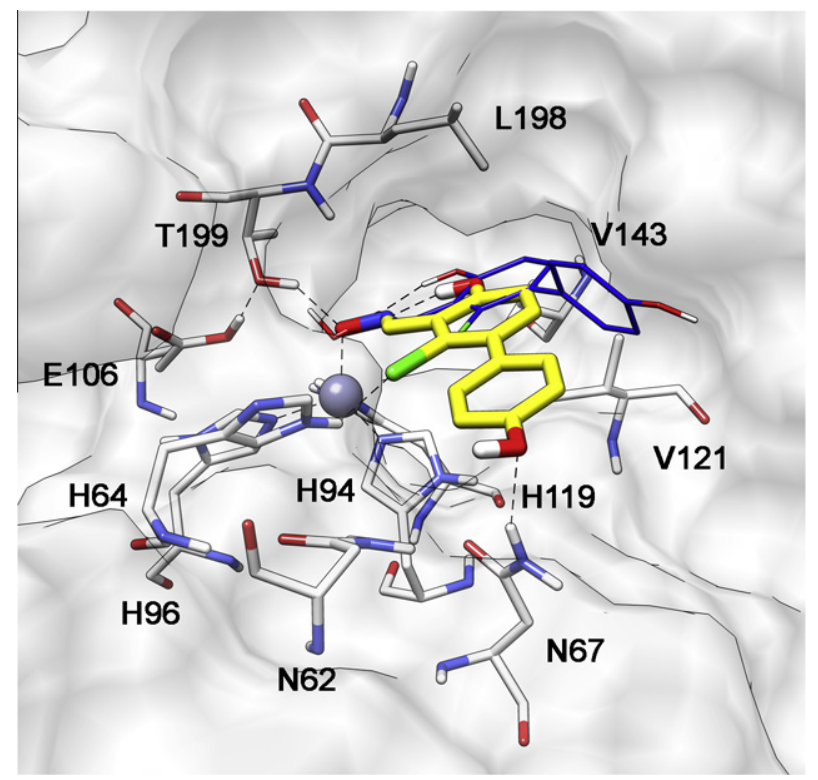

Figure 3. Final structure of the $Q M / M M$ optimization $(6-31++G(d, p)$ basis set). The docking result for compound $\mathbf{2}$ (colored blue) has been reported as a reference structure.

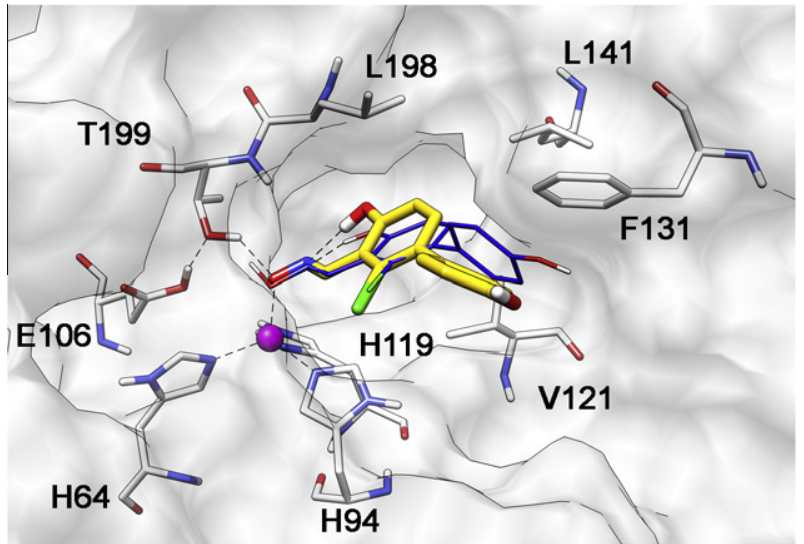

Figure 4. Minimized average structures resulting from the MD simulation of the CAII-2 complex. The docking result for compound $\mathbf{2}$ (colored blue) has been reported as a reference structure.

with the zinc ion and oxime oxygen appeared to be deprotonated with the shift of hydrogen to T199 and the protonation of E106. With regards to the remaining part of the ligand, the 3'-fluoro substituent showed a weak $\mathrm{H}$-bond with $\mathrm{H} 64$ and the oxygen atom of the 4'-hydroxy group of the phenolic substituent formed an $\mathrm{H}$ bond with N67.

\section{5. $\mathrm{QM} / \mathrm{MM}$ dynamic simulation}

As a last step, we further analyzed the CAII-2 complex by means of a $\mathrm{QM} / \mathrm{MM}$ molecular dynamic (MD) simulation. Many authors in the field of drug design have used this approach with good success in finding the correct interactions in biological systems. ${ }^{18,19}$ In particular in our case this approach could be very useful for further confirming the interaction geometry of the ZBG and, most importantly, for analyzing the interactions of the remaining part of the molecule. We used the QM/MM module recently implemented in AMBER $11,{ }^{20}$ by applying the density functional theorybased tight-binding (DFTB) Hamiltonian model in the QM system. ${ }^{21}$ The complex, in an explicit solvent environment, was subjected to two minimization steps and three MD steps, the last one consisting of $4 \mathrm{~ns}$ of QM/MM MD simulation (see the Experimental Section for details). As shown in Figure S1 in the Supplementary Data, the complex seemed to be stable during the simulations. By analyzing the root-mean-square deviation (rmsd) of all the heavy atoms from the X-ray structures, we observed an initial increase due to the equilibration of the system, followed, after 500 ps, by a stabilization of the rmsd value around $1.1 \AA$.

Regarding the geometry of the ligand, we analyzed the rmsd of the position of the ligand with respect to the starting structure during the simulation. During the first $1400 \mathrm{ps}$ the ligand appeared to maintain a disposition very similar to that of the starting structure, then in the last 3600 ps it showed a rmsd value between 0.4 and $0.8 \AA$. This movement of the ligand determined the loss of the two H-bonds with H64 and N67. However, as shown in Figure 4 and Figure S2 in the Supplementary Data, this phenomenon seemed to be determined by the instauration of a favorable $\pi-\pi$ interaction of the aromatic substituent with F131, which strongly stabilizes the ligand interaction. With regards to the ZBG, it showed a disposition which is very similar to the starting docked structure, with the formation of the H-bond with T199.

\section{Conclusions}

A new class of CA inhibitors interacting with the hCAI, II, IX, and XII isoform is herein reported. The salicylaldoxime nucleus 
constitutes a promising novel ZBG to be used in new classes of carbonic anhydrase inhibitors. A computational approach was employed to analyze the binding mode of these new type of inhibitors with carbonic anhydrases and, in particular, to disclose the binding geometry of the ZBG. These findings indicate that the salicylaldoxime scaffold can be considered as a new lead for the development of carbonic anhydrase inhibitors.

\section{Experimental Protocols}

\subsection{CA inhibition}

An Applied Photophysics stopped-flow instrument has been used for assaying the $\mathrm{CA}$ catalysed $\mathrm{CO}_{2}$ hydration activity. ${ }^{22}$ Phenol red (at a concentration of $0.2 \mathrm{mM}$ ) has been used as indicator, working at the maximum absorbance of $557 \mathrm{~nm}$, with $20 \mathrm{mM}$ Hepes ( $\mathrm{pH} 7.5$ ) as buffer, and $20 \mathrm{mM} \mathrm{Na}_{2} \mathrm{SO}_{4}$ (for maintaining constant the ionic strength), following the initial rates of the CA-catalyzed $\mathrm{CO}_{2}$ hydration reaction for a period of $10-$ $100 \mathrm{~s}$. The $\mathrm{CO}_{2}$ concentrations ranged from 1.7 to $17 \mathrm{mM}$ for the determination of the kinetic parameters and inhibition constants. For each inhibitor at least six traces of the initial 5-10\% of the reaction have been used for determining the initial velocity. The uncatalyzed rates were determined in the same manner and subtracted from the total observed rates. Stock solutions of inhibitor $(0.1 \mathrm{mM})$ were prepared in distilled-deionized water and dilutions up to $0.01 \mathrm{nM}$ were done thereafter with distilleddeionized water. Inhibitor and enzyme solutions were preincubated together for $15 \mathrm{~min}-72 \mathrm{~h}$ at room temperature (15 min) or $4{ }^{\circ} \mathrm{C}$ (all other incubation times) prior to assay, in order to allow for the formation of the E-I complex or for the eventual active site mediated hydrolysis of the inhibitor. Data reported in Table 1 show the inhibition after $15 \mathrm{~min}$ incubation, as there were no differences of inhibitory power when the enzyme and inhibitors were kept for longer periods in incubation. ${ }^{23}$ The inhibition constants were obtained by non-linear least-squares methods using PRISM 3, as reported earlier, ${ }^{24}$ and represent the mean from at least three different determinations. The four CA isozymes used in the experiments were recombinant ones, obtained as reported earlier. ${ }^{25-28}$

\subsection{Docking calculations}

Compound 2 was built using Maestro $9.0^{29}$ and it was minimized with Macromodel 9.7. ${ }^{30}$ The conjugated gradient method was applied, until a convergence value of $0.05 \mathrm{~kJ} / \AA$ mol was reached, using the MMFFs force field and a water environment model (generalized-Born/surface-area model), with a distancedependent dielectric constant of 1.0. The minimized ligand was then subjected to a conformational search of 100 steps, using an algorithm based on the Monte Carlo method, with the same force field and parameters used in the minimization. The structure of CAII was extracted from the RCSB Protein Data Bank ${ }^{31}$ (PDB code $\left.3 \mathrm{~V} \mathrm{X}^{16}\right)$. Hydrogen atoms were added by means of Maestro, and the region of interest used by the docking program GOLD version $4.0^{32}$ was defined in order to contain the residues within $15 \AA$ from the original position of the ligand in the X-ray structure. In the docking calculations, the catalytic zinc ion was set to have either a tetrahedral or a trigonal bipyramidal coordination geometry. The 'allow early termination' option was deactivated. The clusterization was set for an rmsd limit of $0.75 \AA$ between the different docking solutions. The remaining GOLD default parameters were used, and the ligand was submitted to 100 genetic algorithm runs by applying the Chemscore fitness scoring function. The clustered structures obtained from the two docking runs were then compared.

\subsection{QM studies}

Geometry optimization was performed by means of quantum mechanical calculations derived from the Gaussian 09 software. ${ }^{33}$ The results of the docking calculations of compound $\mathbf{2}$ into CAII was used as the starting structure. Only the most important atoms of the binding site region were taken into account, that is. the catalytic zinc ion, the imidazole rings of the three His residues surrounding it, the E106 carboxylic group, T199 side chain, and the salicylaldoxime group of compound 2. The QM calculation was carried out using the B3LYP chemical model and two different basis sets (i.e., the LANL2DZ and the $6-31++G(d, p)$ basis set). A direct selfconsistent field (SCF) method with a SCF convergence criterion of $10^{-5}$ was used. The backbone atoms of the residues were kept fixed.

\subsection{QM/MM ONIOM optimization studies}

The ONIOM calculations were carried out using Gaussian 09. ${ }^{33}$ The results of the docking calculations of compound $\mathbf{2}$ into CAII was used as the starting structure. Two layers were considered; the first layer was constituted by the most important atoms of the binding site region were taken into account (i.e. the catalytic zinc ion, the imidazole rings of the three His residues surrounding it, the E106 carboxylic group, T199 side chain, and the salicyladoxime group of compound $\mathbf{2}$ ) whereas the second layer was constituted by the rest of the system. The first layer was analyzed by the B3LYP chemical model and two different basis sets (i.e., the LANL2DZ and the $6-31++G(d, p)$ basis set), whereas the second layer was analyzed through using the MM theory and the AMBER force field. The $\alpha$-Carbon of the residues were kept fixed during the calculations.

\subsection{QM/MM MD calculations}

The calculations were performed using AMBER $11 .^{20} \mathrm{MD}$ simulations were carried out using the parm03 force field at $300 \mathrm{~K}$. The results of the docking calculations of compound $\mathbf{2}$ into CAII was used as starting structure. Compound $\mathbf{2}$ was treated as an anionic ligand, and E106 was considered in the protonated form. The complex was placed in a rectangular parallelepiped water box, an explicit solvent model for water, TIP3P, was used, and the complex was solvated with a $10 \AA$ water cap. Sodium ions were added as counterions to neutralize the system. Prior to the MD, two minimization steps were carried out. In the first stage, the protein was fixed using a harmonic force constant of $500 \mathrm{kcal} / \mathrm{mol} \AA^{2}$ and only the positions of the water molecules were minimized. In the second stage, the entire system was minimized by applying a harmonic force constraint of $15 \mathrm{kcal} / \mathrm{mol} \AA^{2}$ on the $\alpha$-carbons, the zinc ion, and the corresponding coordinating atoms (from the protein and the ligand). The first minimization consisted of 5000 steps with a combined algorithm, namely the sequential use of Steepest Descent (SD) and Conjugate Gradient (CG) methods, for the first 1000 and the last 4000 steps, 'respectively', while the second one consisted of 10,000 steps with 2000 SD and 8000 CG steps. Particle mesh Ewald50 (PME) electrostatics and periodic boundary conditions were used in the simulation. The MD trajectory was conducted using the minimized structure as the starting conformation. The time step of the simulations was $2.0 \mathrm{fs}$ with a cutoff of $10 \AA$ for the nonbonded interaction, and SHAKE was employed to keep rigid all bonds involving hydrogen atoms. Constant volume was carried out for $200 \mathrm{ps,} \mathrm{during} \mathrm{which} \mathrm{the} \mathrm{temperature} \mathrm{was}$ raised from 0 to $300 \mathrm{~K}$ (using the Langevin dynamics method). Next, under constant pressure, an 800 ps MD simulation was carried out at $300 \mathrm{~K}$. During these two MD steps, the same constraints were applied as described for the second minimization step. General Amber force field (GAFF) parameters were assigned to 
the ligands, while partial charges were calculated using the AM1BCC method as implemented in the Antechamber suite of AMBER 11. A 4 ns MD simulation was then performed using the hybrid $\mathrm{QM} / \mathrm{MM}$ method of AMBER 11. The QM region was described by the DFTB theory ${ }^{21}$ and contained the catalytic zinc ion, the imidazole rings of the three His residues surrounding it, the E106 carboxylic group, T199 side chain, and the salicyladoxime group of compound 2. Aside from those atoms within the QM region, the same constraints of the previous MD were applied.

\section{Supplementary data}

Supplementary data associated with this article can be found, in the online version, at http://dx.doi.org/10.1016/j.bmc.2012.08.057.

\section{References and notes}

1. Supuran, C. T. Nat. Rev. Drug. Disc. 2008, 7, 168

2. Supuran, C. T. Bioorg. Med. Chem. Lett. 2010, 20, 3467.

3. Supuran, C. T. Future Med. Chem. 2011, 3, 1165

4. Scozzafava, A.; Mastrolorenzo, A.; Supuran, C. T. Expert Opin. Ther. Pat. 2006, 16, 1627.

5. Schulze Wischeler, J.; Innocenti, A.; Vullo, D.; Agrawal, A.; Cohen, S. M.; Heine, A.; Supuran, C. T.; Klebe, G. ChemMedChem 2010, 5, 1609.

6. Durdagi, S.; Senturk, M.; Ekinci, D.; Balaydin, H. T.; Goksu, S.; Kufrevioglu, O. I.; Innocenti, A.; Scozzafava, A.: Supuran, C. T. Bioorg. Med. Chem. 2011, 19, 1381.

7. Carta, F.; Temperini, C.; Innocenti, A.; Scozzafava, A.; Kaila, K.; Supuran, C. T. J Med. Chem. 2010, 53, 5511

8. Minutolo, F.; Macchia, M.; Katzenellenbogen, B. S.; Katzenellenbogen, J. A. Med. Res. Rev. 2011, 31, 364

9. Tuccinardi, T.; Bertini, S.; Martinelli, A.; Minutolo, F.; Ortore, G.; Placanica, G.; Prota, G.; Rapposelli, S.; Carlson, K. E.; Katzenellenbogen, J. A.; Macchia, M. J Med. Chem. 2006, 49, 5001

10. Minutolo, F.; Bellini, R.; Bertini, S.; Carboni, I.; Lapucci, A.; Pistolesi, L.; Prota, G.; Rapposelli, S.; Solati, F.; Tuccinardi, T.; Martinelli, A.; Stossi, F.; Carlson, K. E.; Katzenellenbogen, B. S.; Katzenellenbogen, J. A.; Macchia, M. J. Med. Chem. 2008, 51, 1344.

11. Minutolo, F.; Bertini, S.; Granchi, C.; Marchitiello, T.; Prota, G.; Rapposelli, S.; Tuccinardi, T.; Martinelli, A.; Gunther, J. R.; Carlson, K. E.; Katzenellenbogen, J A.; Macchia, M. J. Med. Chem. 2009, 52, 858.

12. Bertini, S.; De Cupertinis, A.; Granchi, C.; Bargagli, B.; Tuccinardi, T.; Martinelli, A.; Macchia, M.; Gunther, J. R.; Carlson, K. E.; Katzenellenbogen, J. A.; Minutolo, F. Eur. J. Med. Chem. 2011, 46, 2453.

13. Marques, S. M.; Nuti, E.; Rossello, A.; Supuran, C. T.; Tuccinardi, T.; Martinelli, A.; Santos, M. A. J. Med. Chem. 2008, 51, 7968.

14. Tuccinardi, T.; Nuti, E.; Ortore, G.; Supuran, C. T.; Rossello, A.; Martinelli, A. J. Chem. Inf. Model. 2007, 47, 515.
15. Tuccinardi, T.; Ortore, G.; Rossello, A.; Supuran, C. T.; Martinelli, A. J. Chem. Inf. Model. 2007, 47, 2253.

16. Gitto, R.; Damiano, F. M.; Mader, P.; De Luca, L.; Ferro, S.; Supuran, C. T.; Vullo, D.; Brynda, J.; Rezacova, P.; Chimirri, A. J. Med. Chem. 2012.

17. Vreven, T.; Morokuma, K.; Farkas, O.; Schlegel, H. B.; Frisch, M. J. J. Comput Chem. 2003, 24, 760.

18. Yonezawa, Y.; Nakata, K.; Sakakura, K.; Takada, T.; Nakamura, H. J. Am. Chem. Soc. 2009, 131, 4535.

19. Marques, S. M.; Tuccinardi, T.; Nuti, E.; Santamaria, S.; Andre, V.; Rossello, A.; Martinelli, A.; Santos, M. A. J. Med. Chem. 2011, 54, 8289.

20. Case, D. A.; Darden, T. A.; III, T. E. C.; Simmerling, C. L.; Wang, J.; Duke, R. E.; Luo, R.; Walker, R. C; Zhang, W.; Merz, K. M.; Roberts, B.; Wang, B.; Hayik, S. Roitberg, A.; Seabra, G.; Kolossváry, I.; Wong, K. F.; Paesani, F.; Vanicek, J.; Liu, J.; Wu, X.; Brozell, S. R.; Steinbrecher, T.; Gohlke, H.; Cai, Q.; Ye, X.; Wang, J.; Hsieh, M.-J.; Cui, G.; Roe, D. R.; Mathews, D. H.; Seetin, M. G.; Sagui, C.; Babin, V.; Luchko, T.; Gusarov, S.; Kovalenko, A.; Kollman, P. A.: AMBER, version 11; University of California: San Francisco, CA, 2010.

21. Seifert, G.; Porezag, D.; Frauenheim, T. Int. J. Quantum Chem. 1996, 58, 185

22. Khalifah, R. G. J. Biol. Chem. 1971, 246, 2561.

23. Maresca, A.; Temperini, C.; Vu, H.; Pham, N. B.; Poulsen, S. A.; Scozzafava, A.; Quinn, R. J.; Supuran, C. T. J. Am. Chem. Soc. 2009, 131, 3057.

24. Temperini, C.; Innocenti, A.; Scozzafava, A.; Parkkila, S.; Supuran, C. T. J. Med. Chem. 2010, 53, 850.

25. Alterio, V.; Hilvo, M.; Di Fiore, A.; Supuran, C. T.; Pan, P.; Parkkila, S.; Scaloni, A.; Pastorek, J.; Pastorekova, S.; Pedone, C.; Scozzafava, A.; Monti, S. M.; De Simone, G. Proc. Natl. Acad. Sci. U.S.A. 2009, 106, 16233.

26. Vullo, D.; Innocenti, A.; Nishimori, I.; Pastorek, J.; Scozzafava, A.; Pastorekova, S.; Supuran, C. T. Bioorg. Med. Chem. Lett. 2005, 15, 963.

27. Casey, J. R.; Morgan, P. E.; Vullo, D.; Scozzafava, A.; Mastrolorenzo, A.; Supuran, C. T. J. Med. Chem. 2004, 47, 2337.

28. Casini, A.; Scozzafava, A.; Mincione, F.; Menabuoni, L.; Ilies, M. A.; Supuran, C. T. J. Med. Chem. 2000, 43, 4884.

29. Maestro, version 9.0: Schrödinger Inc: Portland, OR, 2009.

30. Macromodel, version 9.7: Schrödinger Inc: Portland, OR, 2009

31. Berman, H. M.; Westbrook, J.; Feng, Z.; Gilliland, G.; Bhat, T. N.; Weissig, H.; Shindyalov, I. N.; Bourne, P. E. Nucleic Acids Res. 2000, 28, 235.

32. Verdonk, M. L.; Cole, J. C.; Hartshorn, M. J.; Murray, C. W.; Taylor, R. D. Proteins 2003, 52, 609.

33. Frisch, M. J.; Trucks, G. W.; Schlegel, H. B.; Scuseria, G. E.; Robb, M. A.; Cheeseman, J. R.; Scalmani, G.; Barone, V.; Mennucci, B.; Petersson, G. A.; Nakatsuji, H.; Caricato, M.; Li, X.; Hratchian, H. P.; Izmaylov, A. F.; Bloino, J.; Zheng, G.; Sonnenberg, J. L.; Hada, M.; Ehara, M.; Toyota, K.; Fukuda, R.; Hasegawa, J.; Ishida, M.; Nakajima, T.; Honda, Y.; Kitao, O.; Nakai, H.; Vreven, T.; Jr., J. A. M.; Peralta, J. E.; Ogliaro, F.; Bearpark, M.; Heyd, J. J.; Brothers, E.; Kudin, K. N.; Staroverov, V. N.; Kobayashi, R.; Normand, J.; Raghavachari, K.; Rendell, A.; Burant, J. C.; Iyengar, S. S.; Tomasi, J.; Cossi, M.; Rega, N.; Millam, J. M.; Klene, M.; Knox, J. E.; Cross, J. B.; Bakken, V.; Adamo, C.; Jaramillo, J.; Gomperts, R.; Stratmann, R. E.; Yazyev, O.; Austin, A. J.; Cammi, R.; Pomelli, C.; Ochterski, J. W.; Martin, R. L.; Morokuma, K.; Zakrzewski, V. G.; Voth, G. A.; Salvador, P.; Dannenberg, J. J.; Dapprich, S.; Daniels, A. D.; Farkas, Ö.; Foresman, J. B.; Ortiz, J. V.; Cioslowski, J.; Fox, D. J.: Gaussian 09 Revision A.1, Gaussian, Inc., Wallingford CT, 2009. 